\title{
SeaWiFS Lunar Calibration Methodology after Six Years on Orbit
}

\author{
Robert E. Eplee, Jr. ${ }^{a}$, Robert A. Barnes ${ }^{a}$, Frederick S. Patt ${ }^{a}$, \\ Gerhard Meister $^{b}$, and Charles R. McClain ${ }^{c}$ \\ ${ }^{a}$ Science Applications International Corporation, Beltsville, Maryland 20705, USA \\ ${ }^{b}$ Futuretech Corporation, Greenbelt, Maryland 20770, USA \\ ${ }^{c}$ NASA Goddard Space Flight Center, Greenbelt, Mayland 20771, USA
}

\begin{abstract}
The SeaWiFS Project uses monthly lunar calibrations to monitor the on-orbit radiometric stability of SeaWiFS over the course of its mission. Ongoing analyses of the steadily increasing lunar calibration data set have led to improvements in the calibration methodology over time. The lunar measurements must be normalized to a common viewing geometry for the calibration time series to track the radiometric stability of the instrument. Corrections computed from the time and geometry of the observations include Sun-Moon and instrument-Moon distances, oversampling of the lunar image, and variations in the lunar phase angles. The Project has recently implemented a correction for lunar libration that is computed from regressions of the libration angles of the observations against the lunar radiances. Decaying exponential functions of time are fit to the geometry-corrected calibration time series. The observations for bands 1,2, and 5-8 are fit to two simultaneous exponential functions of time, while bands 3 and 4 are fit to single exponential functions of time. The corrections to the radiometric response of the instrument over time are the inverses of these fits. The lunar calibration methodology provides top-of-the-atmosphere radiances for SeaWiFS that are stable to better than $0.07 \%$ over the course of the mission, with residual time drifts that are smaller than $-0.004 \%$ per thousand days. The resulting water-leaving radiances are stable to better than $0.7 \%$, allowing the Project to implement a vicarious calibration of the water-leaving radiances that is independent of time. The calibration methodology presented here will be used to generate the calibration table for the fifth reprocessing of the SeaWiFS global ocean data set.
\end{abstract}

Keywords: SeaWiFS, ocean color, calibration, measurement trends

\section{INTRODUCTION}

SeaWiFS in an eight-band visible and near-infrared scanning radiometer designed to have high radiometric sen* sitivity over oceans without saturating over bright clouds. The SeaWiFS bands are provided in Table 1. The on-orbit calibration strategy for the instrument uses monthly lunar calibrations to monitor the radiometric sta: bility of the individual bands.

For each lunar calibration, the radiances observed by SeaWiFS are integrated over the lunar images. The time series used to monitor the radiometric stability of the instrument are these integrated radiances for each band, normalized by the integrated radiances of the first calibration. The uncorrected time series are shown in Fig. 1. Periodic signals in the time series arise from variations in the geometry of the observations from one lunar calibration to the next. In order to track the radiometric stability of the instrument, the measurements must be normalized to a common viewing geometry. Geometric corrections are computed and applied for the' Sun-Moon and instrument-Moon distances, the oversampling of the lunar images in the along-track direction.

Further author information: (Send correspondence to R.E.E.)

R.E.E.: E-mail: eplee@seawifs.gsfc.nasa.gov, Telephone: 3012860953

R.A.B.: E-mail: rbarnes@seawifs.gsfc.nasa.gov, Telephone: 3012860501

F.S.P.: E-mail: fred@seawifs.gsfc.nasa.gov, Telephone: 3012865723

G.M.: E-mail: meister@simbios.gsfc.nasa.gov, Telephone: 3012860758

C.R.M.: E-mail: Charles.R.McClain@nasa.gov, Telephone: 3012865377 
Table 1. SeaWiFS Bands. The nominal center wavelengths and bandwidths are in nanometers.

\begin{tabular}{|c|c|c|c|c|c|c|c|c|}
\hline Band & $\mathbf{1}$ & $\mathbf{2}$ & $\mathbf{3}$ & $\mathbf{4}$ & $\mathbf{5}$ & $\mathbf{6}$ & $\mathbf{7}$ & $\mathbf{8}$ \\
\hline Wavelength & 412 & 443 & 490 & 510 & 555 & 670 & 765 & 865 \\
\hline Bandwidth & 20 & 20 & 20 & 20 & 20 & 20 & 40 & 40 \\
\hline
\end{tabular}
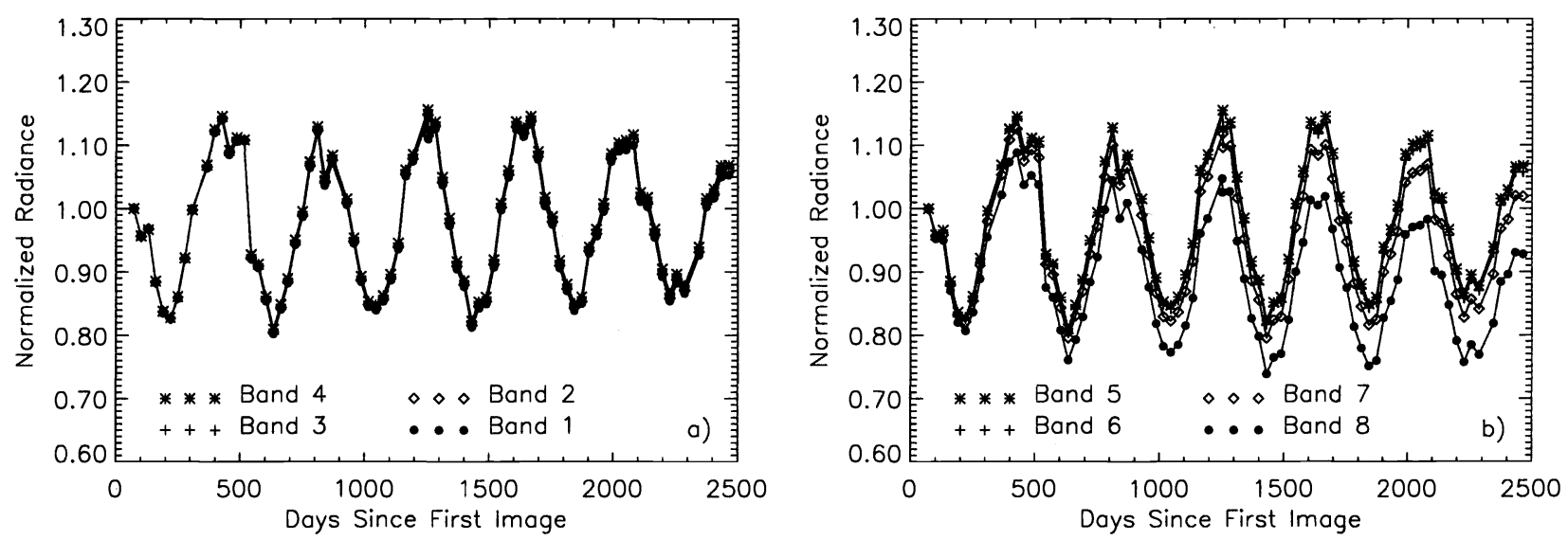

Figure 1. Lunar Calibration Time Series. The periodicities arise from variations in the observing geometry from one calibration to the next.

the phase angles of the observations, and the libration angles of the observations. The current implementations of these corrections for SeaWiFS are improvements on previous geometry correction methodologies. ${ }^{1,2}$

\section{DISTANCE CORRECTIONS}

The distance corrections normalize the observations to a Sun-Moon distance of one Astronomical Unit and to an instrument-Moon distance of one mean Earth-Moon distance. The corrections have the form:

$$
f_{1}(t)=\left(\frac{R_{\text {Sun-Moon }}(t)}{A U}\right)^{2}\left(\frac{R_{\text {Inst-Moon }}(t)}{M L D}\right)^{2}
$$

where:

$\begin{array}{lll}R_{\text {Sun-Moon }} & \equiv \text { Sun-Moon distance } \\ A U & \equiv \text { Astronomical Unit } \\ R_{\text {Inst-Moon }} & \equiv \text { Instrument-Moon distance } \\ M L D & \equiv \text { mean Earth-Moon distance }=384401 \mathrm{~km} \\ t & \equiv \text { time of the observations }\end{array}$

The distance corrections are shown in Fig. 2. The calibration time series with the distance corrections applied are shown in Fig. 3. The distance corrections have removed the large-scale periodic signal from the time series.

\section{OVERSAMPLING CORRECTION}

During lunar calibrations, the spacecraft pitches across the Moon (in the along-track direction) at a slower rate than the scan rate of the instrument, resulting in an oversampled image of the Moon. The oversampling: correction compensates for the pitch rate across the Moon. Since the pitch rate is not known during the lunar calibration maneuvers, the correction is computed by dividing the actual size of the Moon, as seen from the 


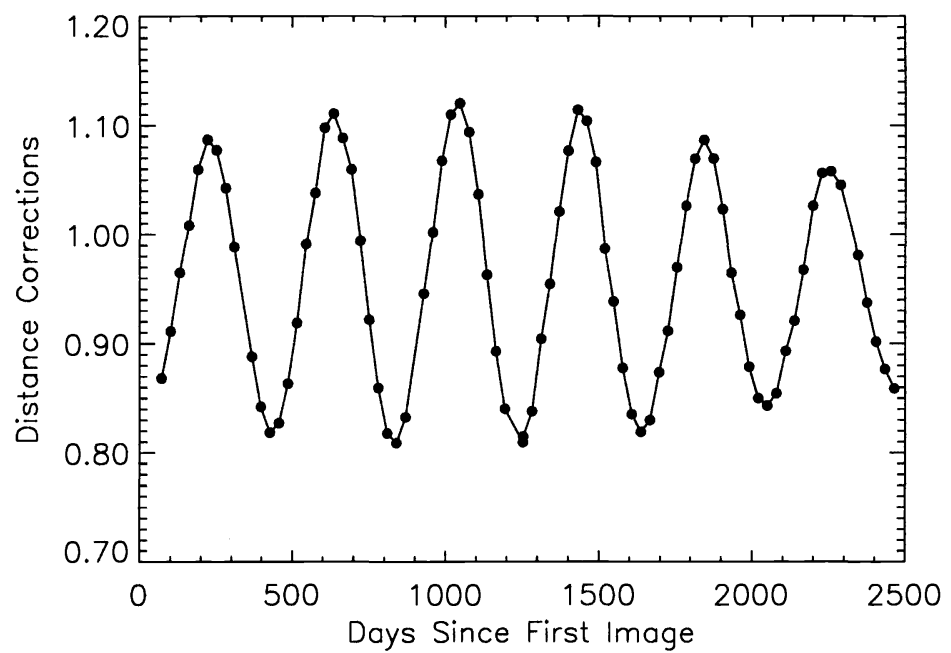

Figure 2. Distance Corrections. These are the combined Sun-Moon and instrument-Moon corrections.
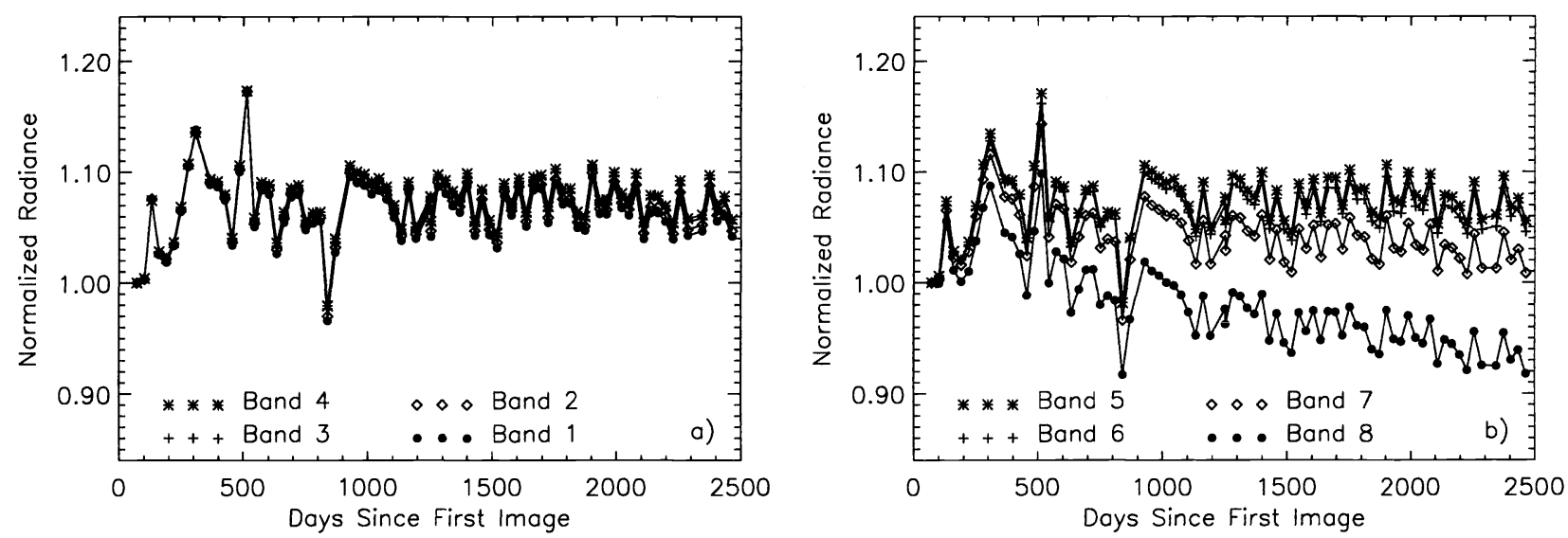

Figure 3. Lunar Calibration Time Series with Distance Corrections. The distance corrections have removed the large-scale periodic signal from the time series. 


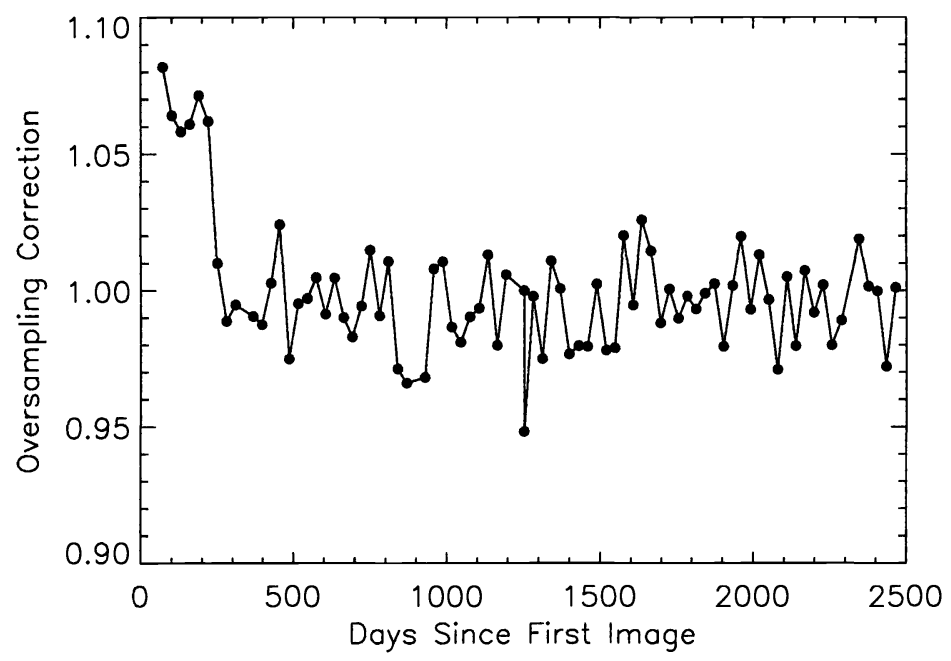

Figure 4. Oversampling Correction. The correction has been normalized to the mean of the time series. The change in the correction between the $6^{\text {th }}$ and $7^{\text {th }}$ calibrations is due to a change in the control gains of the spacecraft attitude control system, which caused a change in the pitch rate of the spacecraft during the lunar calibrations.

spacecraft, by the apparent size of the Moon in the along-track direction in the lunar image. The correction has the form:

$$
f_{2}(t, \alpha, \gamma)=\frac{1}{Y_{M o o n}(\alpha, \gamma)} \arctan \left(\frac{D_{M o o n}}{R_{\text {Inst-Moon }}(t)}\right)
$$

where:

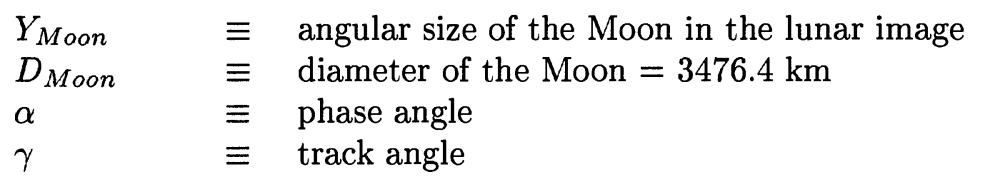

The mean value of the oversampling correction over the SeaWiFS mission is $0.279 \pm 0.007$. This form of the oversampling correction is used when comparing SeaWiFS lunar observations with external observations or models. The concern in monitoring the radiometric stability of the instrument is variations in the oversampling from calibration to calibration. So, the oversampling correction is normalized by the mean of the correction over the mission, yielding a correction with a mean value of unity over the the mission. This correction is shown in Fig. 4 . The change in the correction between the $6^{\text {th }}$ and $7^{\text {th }}$ calibrations is due to a change in the control gains of the spacecraft attitude control system in May of 1998, which caused a change in the pitch rate of the spacecraft during the lunar calibrations. The calibration time series with the oversampling correction applied are shown in Fig. 5. The oversampling correction reduces some of the high-frequency periodic signal in the time series and reduces the scatter in the first six calibrations that was caused by the initial gains of the attitude control system.

\subsection{Lunar Image Size Determination}

The size of the Moon in the lunar images is the observational input into the oversampling correction. This parameter is derived from the $2^{\text {nd }}$ derivative of the radiance profile across the Moon. The edge locations of each profile are determined from the first maximum and first minimum of the profile coming from the off-of-the-Moon direction. The first maximum is the first pixel where the edge of the Moon is detected within the pixel and the first minimum is the first pixel where the Moon fills the pixel completely. The edge location is defined to be the point where the radiance is $\frac{1}{2}$ of the difference in the radiances of the pixels at the first maximum and first minimum. The two edges of the profile are determined from the appropriate maximum and minimum. For each lunar image, the size is computed for each profile across the Moon in the along-track direction. Since the central profile across the Moon cannot be identified from the navigation data, the size for the image is defined 

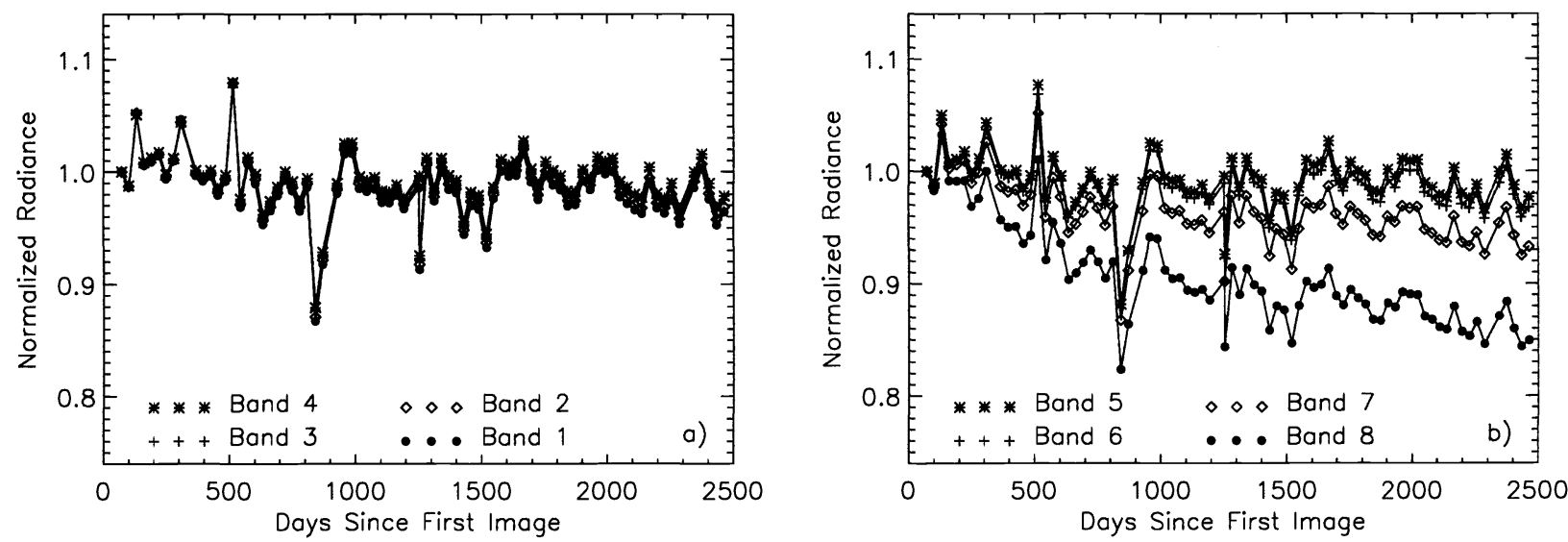

Figure 5. Lunar Calibration Time Series with Oversampling Correction. The oversampling correction has reduced some of the the high-frequency periodic signal from the time series and has reduced the scatter in the first six calibrations.

to be the maximum size from the various profiles. For a given lunar calibration, the size of the Moon used in the oversampling correction is the mean of the sizes determined for all eight bands. The standard deviation of this band-to-band mean, over the course of the mission, is 0.18 pixels. This is one estimate of the uncertainty in the determination of the size of the lunar image.

The size of the Moon determined from this approach is also dependent on the track angle, the angle between the along-track direction of the spacecraft field-of-view during the calibration maneuver and the rotational axis of the Moon. A track that is not along the rotational axis of the Moon could intersect the lunar terminator rather than the edge of the Moon, yielding an underestimate of the actual size of the Moon in the lunar image: A correction for this underestimation is dependent on both the track angle and on the phase angle of the lunar image (which determines the location of the terminator). The reduction in the image size due to the track angle and the phase angle is:

$$
r_{\text {track }}(\alpha, \gamma)=\frac{\cos \alpha}{\sqrt{1-(1+\cos \alpha)(1-\cos \alpha)(\cos \gamma)^{2}}}
$$

where:

$$
\begin{aligned}
& \alpha \equiv \text { phase angle } \\
& \gamma \equiv \text { track angle }
\end{aligned}
$$

The size of the Moon, corrected for the track angle, is:

$$
Y_{\text {Moon }}(\alpha, \gamma)=\frac{2}{r_{\text {track }}(\alpha, \gamma)+1} Y_{o b s}
$$

where $Y_{o b s} \equiv$ observed size of the Moon. The track-angle corrections for the lunar calibration time series have values of 1.0-1.0034, with a mean of 1.0013. The corrected size of the Moon is used in computing the oversampling correction.

\section{PHASE ANGLE CORRECTIONS}

The phase angle corrections normalize the observations to a lunar phase angle of $7^{\circ}$. This phase angle was chosen for the calibration measurements to maximize the illuminated surface of the Moon while minimizing the opposition effect, i.e., the increase in brightness of sunlight diffusely reflected from a particulate surface near zero phase. The change in the overall reflectance of the lunar surface with phase angle is non-Lambertian and arises from changes in both the reflectance of the surface and the area of the surface illuminated by the sun 

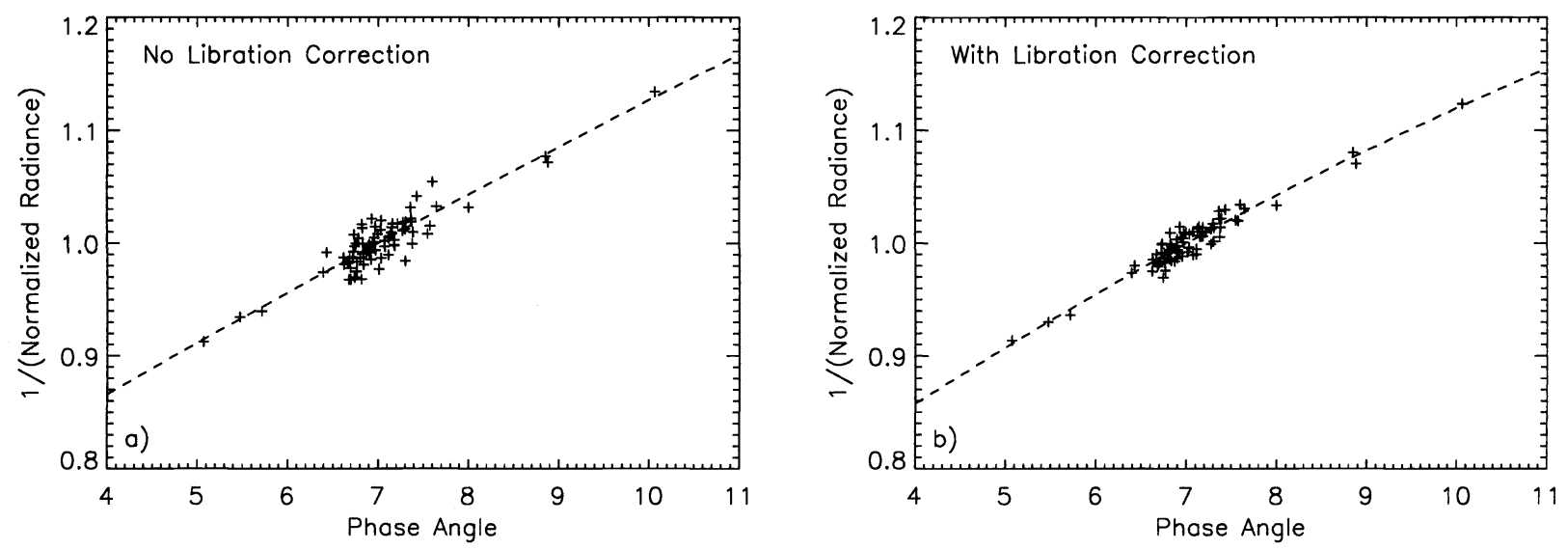

Figure 6. Phase Dependencies for Band 1. The scatter in a) is due to libration, which has been corrected in b).

Operational considerations have caused the lunar calibrations to occur over a phase angle range of $5^{\circ}-10^{\circ}$. Of the 79 lunar calibrations that have occurred to date, three took place at phase angles of less than $6^{\circ}$, three took place at phase angles of more than $8^{\circ}$, and the median phase angle is $6.95^{\circ}$.

To compute the correction for each band, a quadratic function of the phase angle has been fit to the inverse of the lunar time series over a phase angle range of $4^{\circ}-11^{\circ}$. These fits have been normalized to yield values of unity at a phase angle of $7^{\circ}$. The fit for Band 1 is shown in Fig. 6 a; this behavior is typical of the other bands. The scatter in the data arises because the lunar albedo is not symmetric about $0^{\circ}$ phase. Thirty-one of the lunar calibrations, including the three below $6^{\circ}$ and the three above $8^{\circ}$, occurred before full phase and 48 of the calibrations occurred after full phase. To reduce the scatter in the data and improve the computation of the phase correction coefficients, libration corrections (discussed in the next section) were computed for the phase-corrected calibration time series. These libration corrections were applied to the normalized lunar radiances and the phase angle corrections were recomputed. The revised fit for Band 1 is shown in Fig. 6 b; again, this behavior is typical of the other bands. For each band, the scatter in the data have been significantly reduced by the libration corrections. Phase corrections derived from the libration-corrected fits are used to process the lunar calibration time series.

The phase corrections applied to the calibration time series have the form:

$$
f_{3}(\lambda, \alpha)=\left(p_{0}(\lambda)+p_{1}(\lambda) \alpha+p_{2}(\lambda) \alpha^{2}\right)
$$

where:

$$
\begin{aligned}
& p_{0} \equiv \text { constant term of the correction } \\
& p_{1} \equiv \text { linear term of the correction } \\
& p_{2} \equiv \text { quadratic term of the correction } \\
& \lambda \equiv \text { SeaWiFS band }
\end{aligned}
$$

The phase angles and corrections for Band 1 are shown in Fig. 7. The corrections become significant as the phase angle of the calibrations depart from $7^{\circ}$.

The lunar calibration time series with the distance corrections, the oversampling correction, and the phase angle corrections applied have the form:

$$
\frac{S_{1}(t, \lambda, \alpha, \gamma)}{S_{1}\left(t_{i}, \lambda, \alpha, \gamma\right)}=\frac{S_{o b s}(t, \lambda, \alpha, \gamma)}{S_{o b s}\left(t_{i}, \lambda, \alpha, \gamma\right)} f_{1}(t) f_{2}(t, \alpha, \gamma) f_{3}(\lambda, \alpha)
$$

where: 

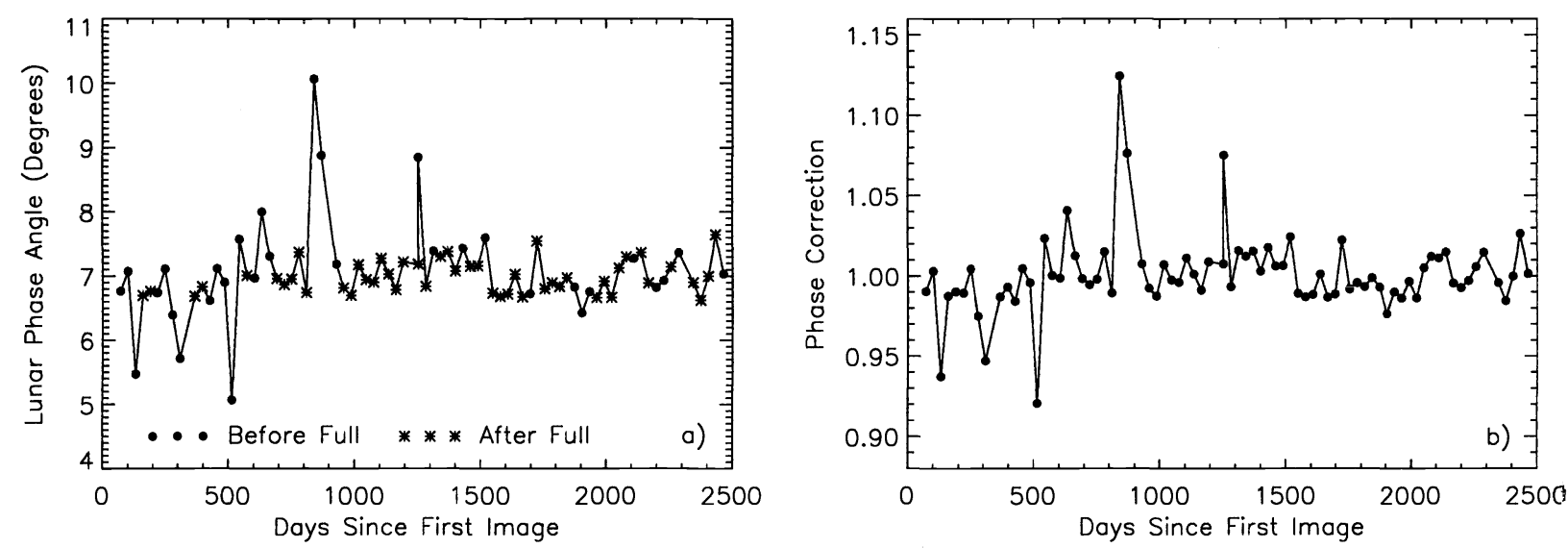

Figure 7. Phase Angles and Corrections for Band 1. The calibrations before and after full phase are shown in a). As is shown in b), the corrections grow in magnitude as the phase angle of the calibrations depart from $7^{\circ}$.
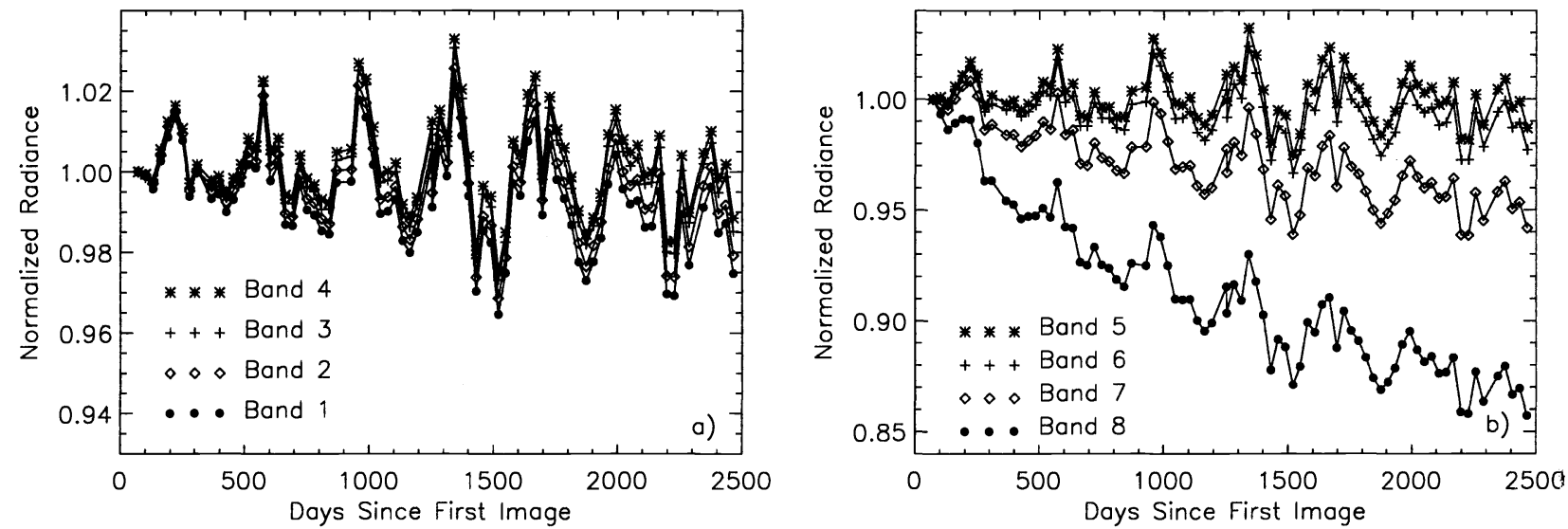

Figure 8. Phase-Corrected Lunar Calibration Time Series. The dominant periodic signal remaining in the time series is libration.

$$
\begin{aligned}
& S_{1} \equiv \text { phase-corrected integrated radiances } \\
& S_{\text {obs }} \equiv \text { observed integrated radiances } \\
& t_{i} \equiv \text { time of the first lunar calibration }
\end{aligned}
$$

These phase-corrected time series are shown in Fig. 8. The dominant periodic signal remaining in the time series is libration.

\section{LIBRATION CORRECTIONS}

Libration effects are changes in the lunar radiance as seen from the spacecraft due to variation in the side of the Moon that faces the Earth during the lunar observations (the libration of the Moon). Commonly, the libration angles are defined to be the selenographic longitude and latitude of the sub-Earth point on the lunar surface. For correcting the lunar calibration time series, the libration angles are defined to be the coordinates of the sub-spacecraft point on the lunar surface. Since the amount of sunlight reflected by the lunar surface is a function of the position of the sun in the lunar sky, the definition of the libration angles is expanded to include the selenographic coordinates of the sub-solar points on the lunar surface. These four sets of coordinates constitute the libration angles for computing the libration effects in the SeaWiFS data. The time series of the libration angles are shown in Fig. 9. 

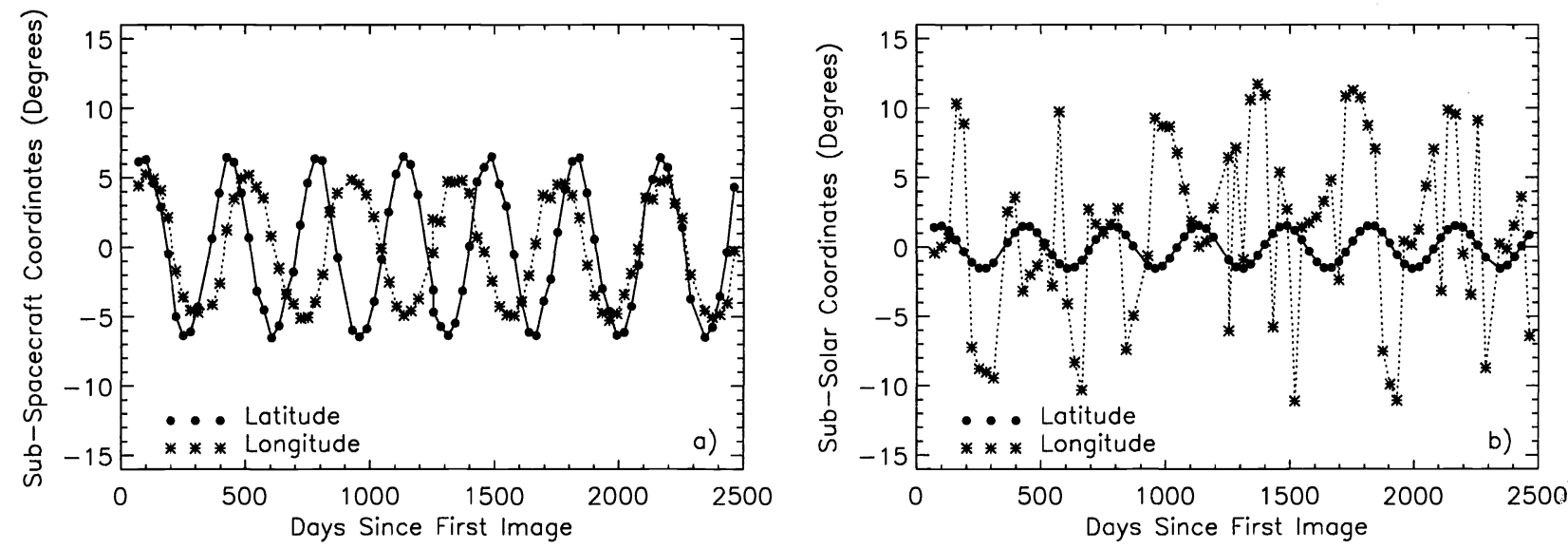

Figure 9. Libration Angles. a) shows the sub-spacecraft angles and b) shows the sub-solar angles.

The libration effects in the SeaWiFS data are computed from linear regressions of the libration angles against the phase-corrected lunar calibration time series for each band. The regression fits have the form:

$$
\left(c_{0}+c_{1} l_{s c}+c_{2} b_{s c}+c_{3} l_{s u n}+c_{4} b_{s u n}\right)=\frac{S_{1}(t, \lambda, \alpha, \gamma)}{S_{1}\left(t_{i}, \lambda, \alpha, \gamma\right)}
$$

where:

$c_{0} \equiv$ libration correction constant
$c_{1} \equiv$ libration sub-spacecraft longitude correction coefficient
$c_{2} \equiv$ libration sub-spacecraft latitude correction coefficient
$c_{3} \equiv$ libration sub-solar longitude correction coefficient
$c_{4} \equiv$ libration sub-solar latitude correction coefficient
$l_{s c} \equiv$ longitude of the sub-spacecraft point
$b_{s c} \equiv$ latitude of the sub-spacecraft point
$l_{\text {sun }} \equiv$ longitude of the sub-solar point
$b_{\text {sun }} \equiv$ latitude of the sub-solar point

The libration corrections that are applied to the calibration time series are the inverse of the libration effects:

$$
f_{4}\left(l_{s c}, b_{s c}, l_{s u n}, b_{s u n}\right)=\left(c_{0}+c_{1} l_{s c}+c_{2} b_{s c}+c_{3} l_{\text {sun }}+c_{4} b_{s u n}\right)^{-1}
$$

The libration corrections should have a minimal wavelength dependence, if any. However, the linear regression approach to computing the libration effects breaks down for bands where the change in the radiometric response of the instrument with time is comparable in magnitude to the libration effects. Such conditions occur for bands 7 and 8. In order to obtain the most accurate libration corrections possible the libration effects for bands 4 and 5 , which have the smallest change with time, are averaged on a calibration-by-calibration basis. These mean libration effects are shown in the Fig. 10. The libration corrections, the inverse of the mean libration effects for Bands 4 and 5, are applied to all eight bands for the lunar calibration time series analysis.

The application of the libration corrections to the phase-corrected calibration time series yields a time series which has the form:

$$
\frac{S_{2}\left(t, \lambda, \alpha, \gamma, l_{s c}, b_{s c}, l_{s u n}, b_{s u n}\right)}{S_{2}\left(t_{i}, \lambda, \alpha, \gamma, l_{s c}, b_{s c}, l_{s u n}, b_{s u n}\right)}=\frac{S_{1}(t, \lambda, \alpha, \gamma)}{S_{1}\left(t_{i}, \lambda, \alpha, \gamma\right)} f_{4}\left(l_{s c}, b_{s c}, l_{s u n}, b_{s u n}\right)
$$

where $S_{2} \equiv$ libration-corrected integrated radiances. The time series is shown in Fig. 11. 


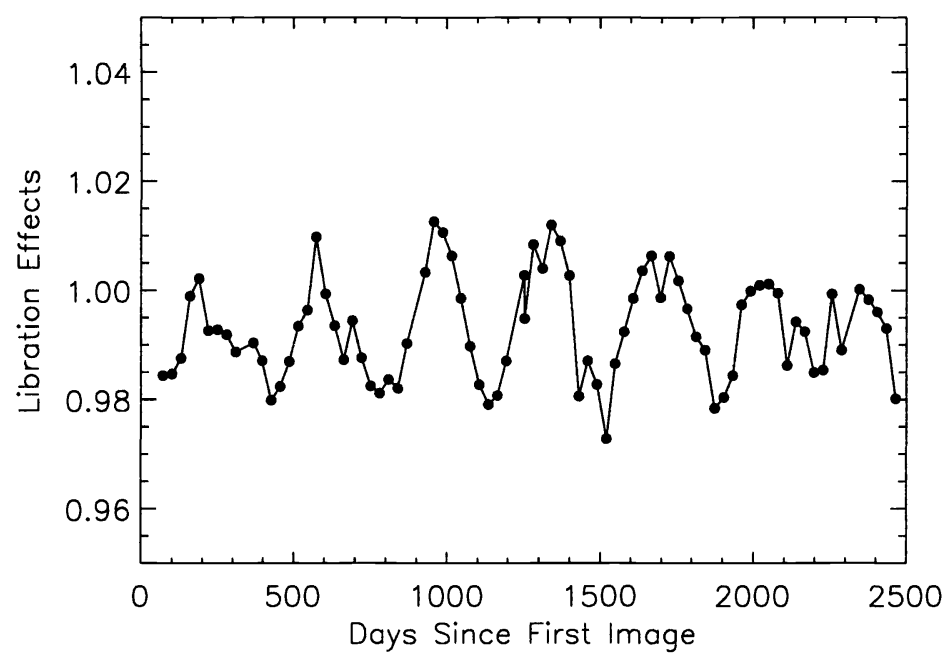

Figure 10. Libration Effects. The libration effects are independent of wavelength.
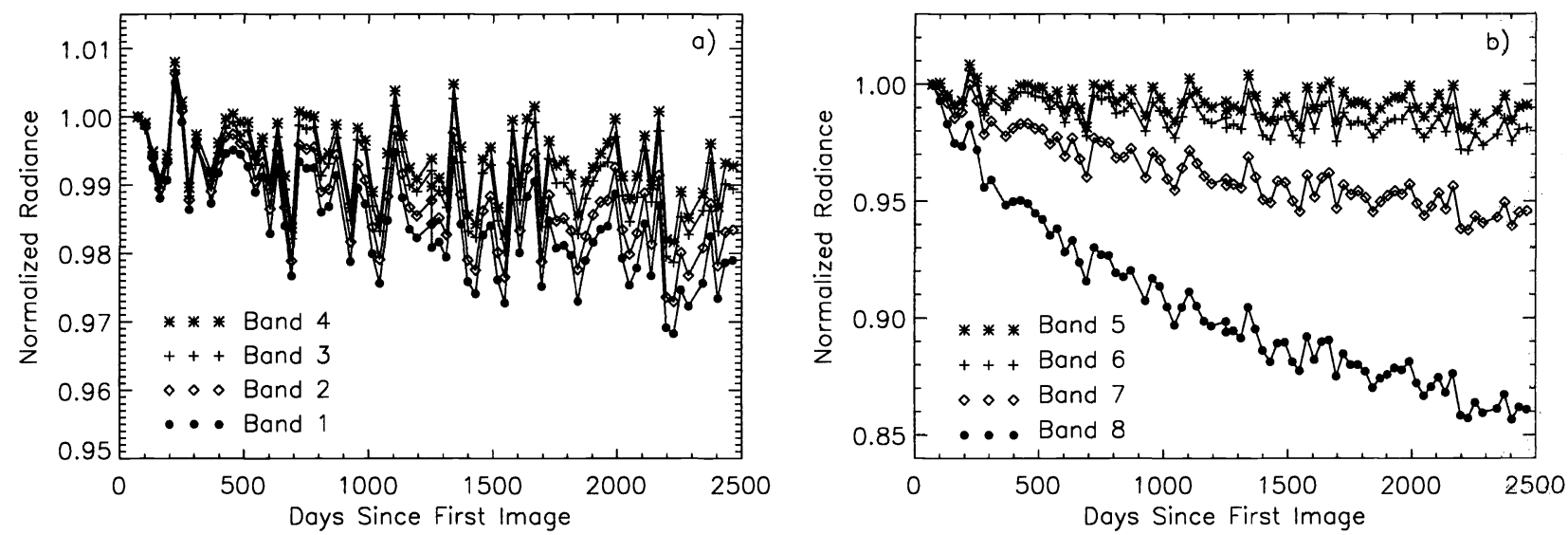

Figure 11. Libration-Corrected Lunar Calibration Time Series. The plots show a systematic noise that is correlated across all eight bands. 


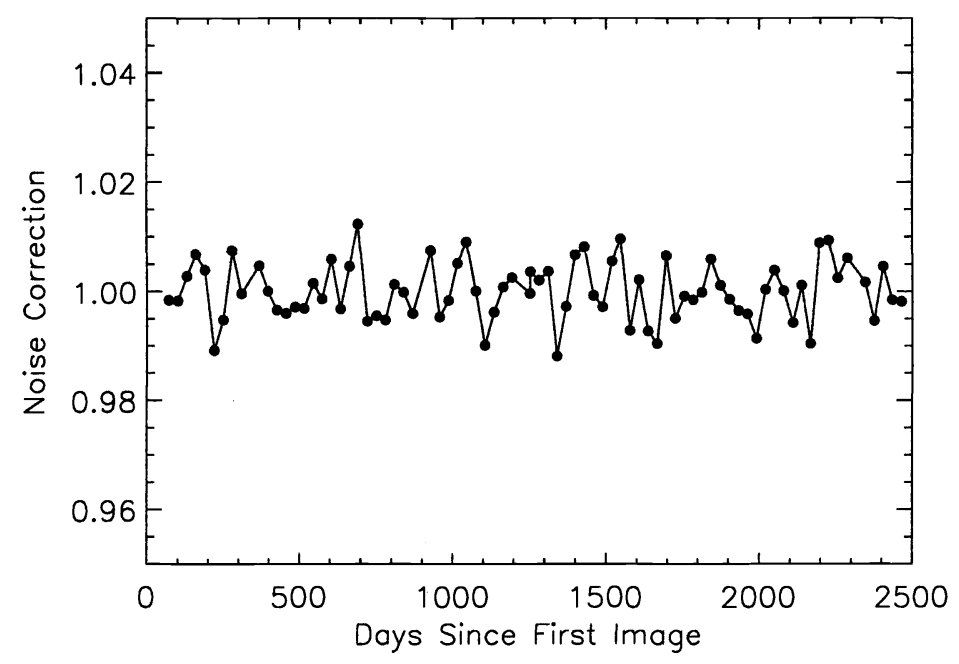

Figure 12. Noise Reduction Correction. This correction removes fixed-pattern noise common to all eight bands.

\section{NOISE REDUCTION CORRECTION}

The libration-corrected lunar calibration time series show a noise that is correlated across all eight bands. This noise is a systematic error in the observations that is common to all of the bands. The primary systematic error is the uncertainty in the determination of the size of the Moon used in computing the oversampling correction.

An estimate of the correlated noise for each band can be obtained by computing the residuals of an exponential fit to the libration-corrected calibration time series. A noise reduction correction can be computed from these residuals and applied to the time series. The correction should not change any time-dependences in the radiometric response of a given band. The correction has the form:

$$
f_{5}(t, \lambda)=1-\frac{\frac{S_{2}\left(t, \lambda, \alpha, \gamma, l_{s c}, b_{s c}, l_{s u n}, b_{s u n}\right)}{S_{2}\left(t_{i}, \lambda, \alpha, \gamma, l_{s c}, b_{s c}, l_{s u n}, b_{s u n}\right)}-C(t, \lambda)}{C(t, \lambda)}
$$

where $C(t, \lambda) \equiv$ exponential fit to the libration-corrected time series. A single exponential with a 1600 day time constant is used for the fits. The determination of the time constant is discussed in the next section. Since bands 4 and 5 show the smallest change with time, the noise reduction correction from the mean of the noise residuals for these bands will be applied to all eight bands. This correction is shown in Fig. 12.

\section{LUNAR CALIBRATION TIME SERIES}

The fully-corrected lunar radiances have had the distance, oversampling, phase angle, libration, and noise reduction corrections applied. The resulting time series has the form:

$$
\frac{S\left(t, \lambda, \alpha, \gamma, l_{s c}, b_{s c}, l_{s u n}, b_{\text {sun }}\right)}{S\left(t_{i}, \lambda, \alpha, \gamma, l_{s c}, b_{s c}, l_{\text {sun }}, b_{\text {sun }}\right)}=\frac{S_{2}\left(t, \lambda, \alpha, \gamma, l_{s c}, b_{s c}, l_{\text {sun }}, b_{\text {sun }}\right)}{S_{2}\left(t_{i}, \lambda, \alpha, \gamma, l_{s c}, b_{s c}, l_{s u n}, b_{\text {sun }}\right)} f_{5}(t, \lambda)
$$

where $S \equiv$ corrected integrated radiances. The corrected lunar calibration time series are shown in Fig. 13. Examination of the time series shows that the periodic geometric effects and the systematic noise effects have been removed from the time series.

The lunar calibration time series can be fit by a decaying exponential function of time for each band. The time series for bands 1,2 ,and 5-8 are fit by two simultaneous exponential functions of time, while bands 3 and 4 

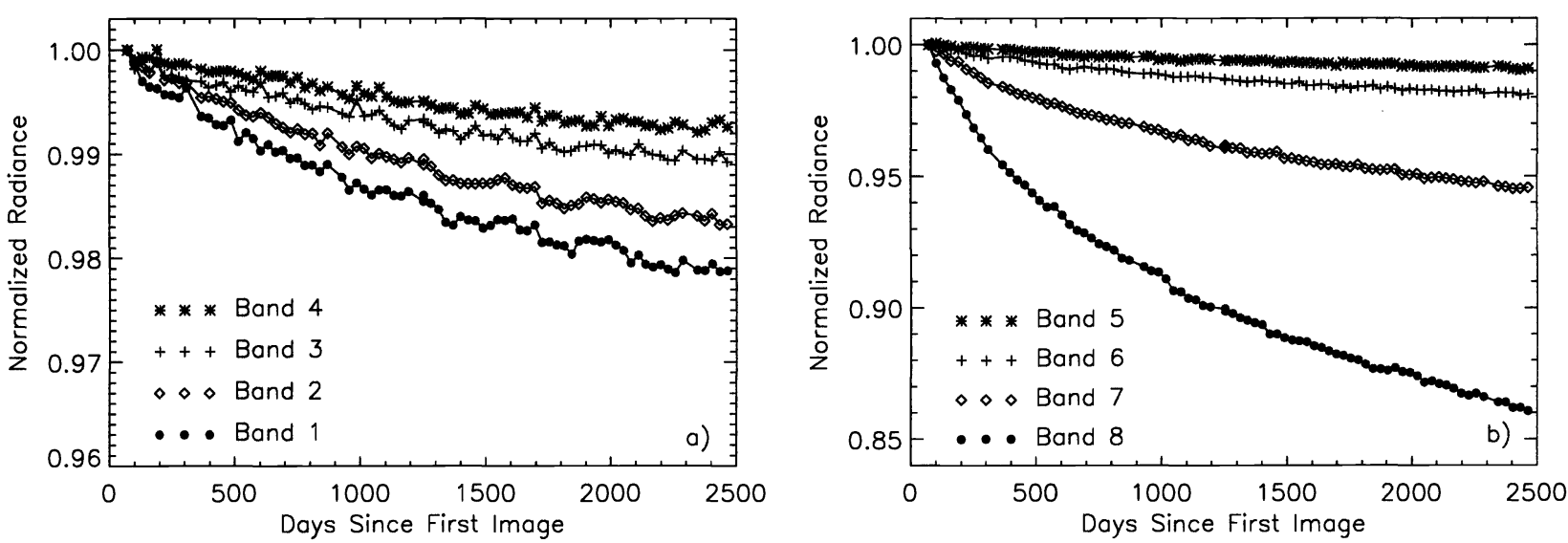

Figure 13. SeaWiFS Lunar Calibration Time Series. The periodic geometric effects and the systematic noise effects have been removed.

are fit by single exponential functions of time. These functions have the form:

$$
f(\lambda, t)=a_{0}(\lambda)-a_{1}(\lambda)\left[1-e^{-C_{1}(\lambda)\left(t-t_{0}\right)}\right]-a_{2}(\lambda)\left[1-e^{-C_{2}(\lambda)\left(t-t_{0}\right)}\right]
$$

where:

$a_{0} \equiv$ the initial value of the function
$a_{1} \equiv$ amplitude of first exponential function
$a_{2} \equiv$ amplitude of second exponential function
$C_{1} \equiv$ time constant of first exponential function
$C_{2} \equiv$ time constant of second exponential function
$t \equiv$ the time of the observation
$t_{0} \equiv$ the reference time for the time series

The time constants for the fits were derived from separate single-exponential fits to the time series for the early and late stages of the mission. For the simultaneous exponentials, the time series are fit with a short period time constant of 200 days and with a long period time constant of 1600 days. For the single exponential, the time series are fit with the long period, 1600 day time constant.

The fits to the calibration time series are shown in Fig. 14. These fits will be incorporated into the calibration table used for the fifth reprocessing of the SeaWiFS global ocean data set.

Two different decay mechanisms are responsible for the changes in response of each of the bands. The first mechanism caused a rapid decrease in response that decayed away after approximately the first year of the mission. This effect is probably present in bands 3 and 4, though with a magnitude that is not detectable in the scatter of the observations. There is no current candidate for this short-term mechanism. The second mechanism has been in effect over the entire mission. The long-period decay for the shorter wavelength bands (bands 1-4) most likely arises from yellowing of the instrument optics on orbit. The long-period decay for the longer wavelength bands (bands 5-8) most likely arises from charged-particle induced damage to the silicon photodiodes.

\section{CALIBRATION ASSESSMENT}

As an evaluation of the radiometric corrections over time, the lunar data have been calibrated with the exponential functions described above. These calibrated time series have been processed through the geometric corrections described in this paper. The time series for Band 5 is shown in Fig. 15; this time series is typical of the other bands. The first plot shows the libration-corrected time series. The second plot shows the time serie, 

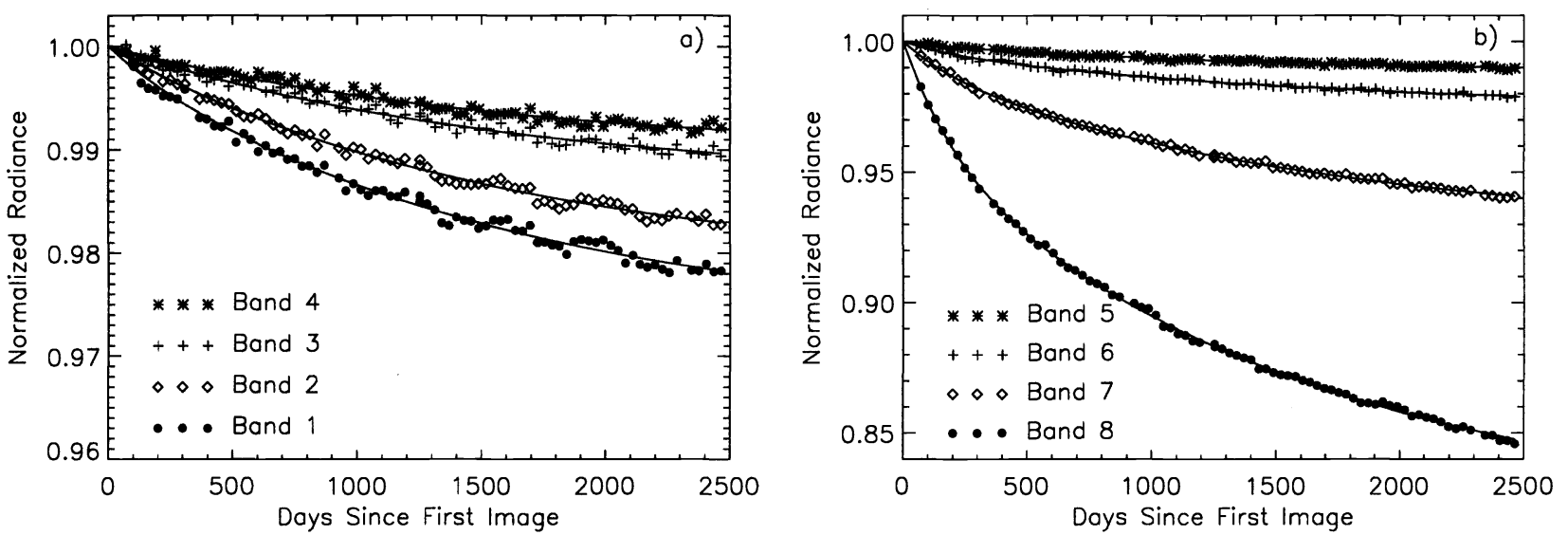

Figure 14. Fits to the Lunar Calibration Time Series. The exponential fits have been applied to the geometrycorrected time series.

after the noise correction is applied.

As a validation of the radiometric corrections over time, the calibrated lunar data have been processed through the USGS lunar model developed for remote sensing instrument calibration. ${ }^{3,4}$ The USGS lunar model provides an alternate approach to the geometric corrections used by the SeaWiFS Project. The time series for Band 5 is also shown in Fig. 15; this time series is typical of the other bands when processed through the USGS model. A noise reduction correction was computed for the data processed through USGS model and applied in the second plot.

Examination of the plots in Fig. 15 shows that the SeaWiFS geometric corrections and the USGS lunar model yield results that are equivalent to within the scatter in the observations. The differences arise from the respective approaches to the geometric corrections implemented by the SeaWiFS Project and by the USGS lunar model. The primary source of random errors in the time series is measurement error. The primary source of systematic errors in the time series are the uncertainties in the oversampling correction; the USGS lunar mode] uses the size of the Moon as determined from the images as input, so these uncertainties are present in the USGS model output as well. The radiances in the calibrated lunar time series are stable to better than $0.07 \%$ over the 2500-day course of the mission, as derived from the SeaWiFS geometric corrections and as confirmed from the USGS lunar model output. The residual drifts in the radiometric response of the instrument over time are reduced by factors of 100 , and are typically smaller than $-0.004 \%$ per thousand days. These results confirm previous comparisons between calibrated SeaWiFS lunar radiances and the USGS lunar model. ${ }^{5}$

These results show that the SeaWiFS lunar calibration methodology provides top-of-the-atmosphere radiances for SeaWiFS that are stable to better than $0.07 \%$ over the mission. The resulting water-leaving radiances are stable to better than $0.7 \%$, allowing the SeaWiFS Project to implement a vicarious calibration of the waterleaving radiances that is independent of time.

Finally, the calibration methodologies reported in this paper monitor the relative change in the radiometric response of the instrument on orbit. The full calibration of the instrument requires knowledge of the radiometric response of the instrument at the start of on-orbit operations. The initial response of the instrument was provided by the prelaunch calibration of the instrument. ${ }^{6}$ Limits on changes in the instrument response during launch were derived from the SeaWiFS transfer-to-orbit experiment. ${ }^{7,8}$ Taken together, the prelaunch calibration, the transfer-to-orbit, and the on-orbit lunar calibrations provide the stable top-of-the-atmosphere radiances required for the accurate retrievals of water-leaving radiances from the SeaWiFS ocean data. 

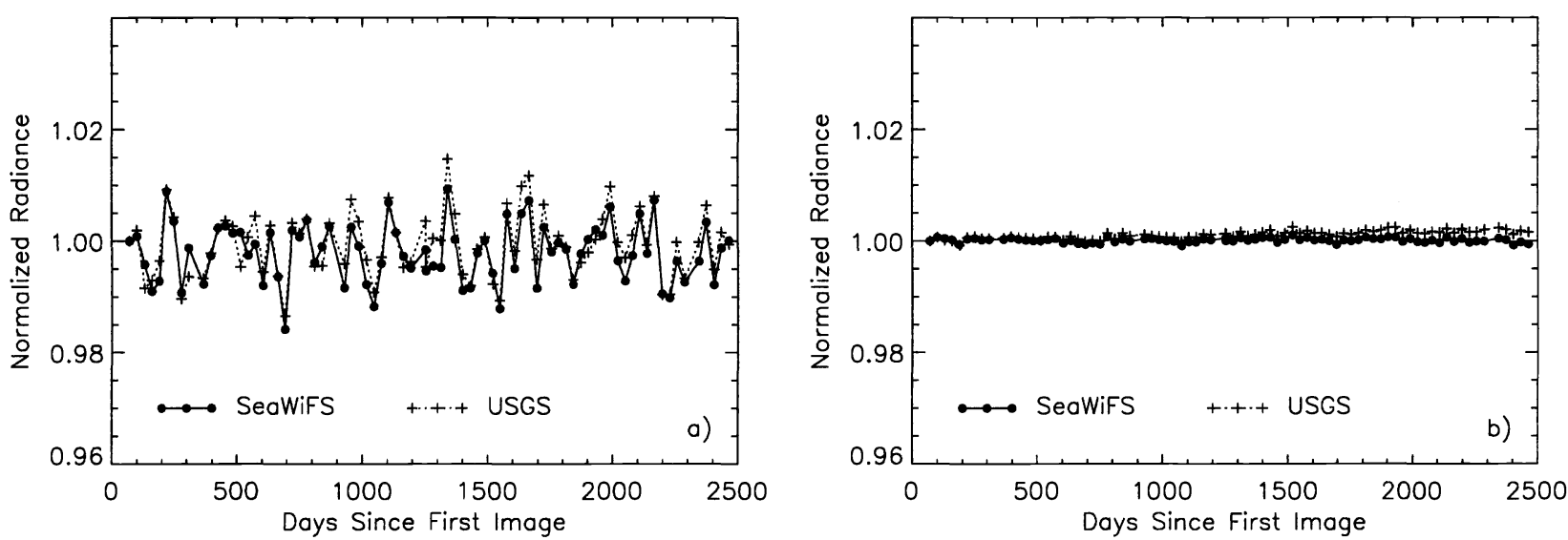

Figure 15. Calibrated Lunar Time Series for Band 5. a) shows the libration-corrected time series (SeaWiFS) and the USGS model output. b) shows the two time series after the noise reduction corrections have been applied. The time series are equivalent to within the scatter in the observations.

\section{REFERENCES}

1. R.A. Barnes, R.E. Eplee, Jr., F.S. Patt, and C.R. McClain, "Changes in the radiometric stability of SeaWiFS determined from lunar and solar-based measurements," Appl. Opt. 38, pp. 4649-4664, 1999.

2. R.A. Barnes, R.E. Eplee, Jr., G.M. Schmidt, F.S. Patt, and C.R. McClain, "Calibration of SeaWiFS. I. Direct techniques," Appl. Opt. 40, pp. 6682-6670, 2001.

3. T.C. Stone and H.H. Kieffer, "Absolute irradiance of the Moon for on-orbit calibration," in Earth Observing Systems VII, W.L. Barnes, ed., Proc. SPIE 4814, pp. 211-221, 2002.

4. H.H. Kieffer, T.C. Stone, R.A. Barnes, S. Bender, R.E. Eplee, Jr., J. Mendenhall, and L. Ong, "On-orbit radiometric calibration over time and between spacecraft using the Moon", in Sensors, Systems, and Generation Next-Generation Satellites VI, H. Fujisada, J.B. Lurie, M.L. Aten, and K. Weber, eds., Proc. SPIE 4881, pp. 287-298, 2003.

5. R.A. Barnes, R.E. Eplee, Jr., F.S. Patt, H.H. Kieffer, T.C. Stone, G. Meister, J.J. Butler, and C.R. McClain, "Comparison of the on-orbit response history of SeaWiFS with the USGS lunar model," Appl. Opt., in press, 2004.

6. B.C. Johnson, E.A. Early, R.E. Eplee, Jr., R.A. Barnes, and R.T. Caffrey, The 1997 Prelaunch Radiometric Calibration of SeaWiFS, NASA Tech. Memo. 1999-206892 4, S.B. Hooker and E.R. Firestone, eds., (NASA Goddard Space Flight Center, Greenbelt, Maryland), 51 pp., 1999.

7. R.A. Barnes, R.E. Eplee, Jr., S.F. Biggar, K.J. Thome, E.F. Zalewski, P.N. Slater, and A.W. Holmes, "SeaWiFS transfer-to-orbit experiment," Appl. Opt. 39, pp. 5620-5631, 2000.

8. R.A. Barnes and E.F. Zalewski, "Reflectance-based calibration of SeaWiFS. II. Conversion to radiance," Appl. Opt. 42, pp. 1648-1660, 2003. 